Sistemas lineares, aplicações e uma sequência didática 



\title{
Sistemas lineares, aplicações e uma sequência didática
}

\author{
Sônia Aparecida Carreira Rufato
}

Orientadora: Profa. Dra.Ires Dias

Dissertação apresentada ao Instituto de Ciências Matemáticas e de Computação - ICMC-USP, como parte dos requisitos para obtenção do título de "Mestre - Programa de Mestrado Profissional em Matemática." VERSÃO REVISADA.

USP - São Carlos

Janeiro de 2014 
Ficha catalográfica elaborada pela Biblioteca Prof. Achille Bassi e Seção Técnica de Informática, ICMC/USP, com os dados fornecidos pelo(a) autor(a)

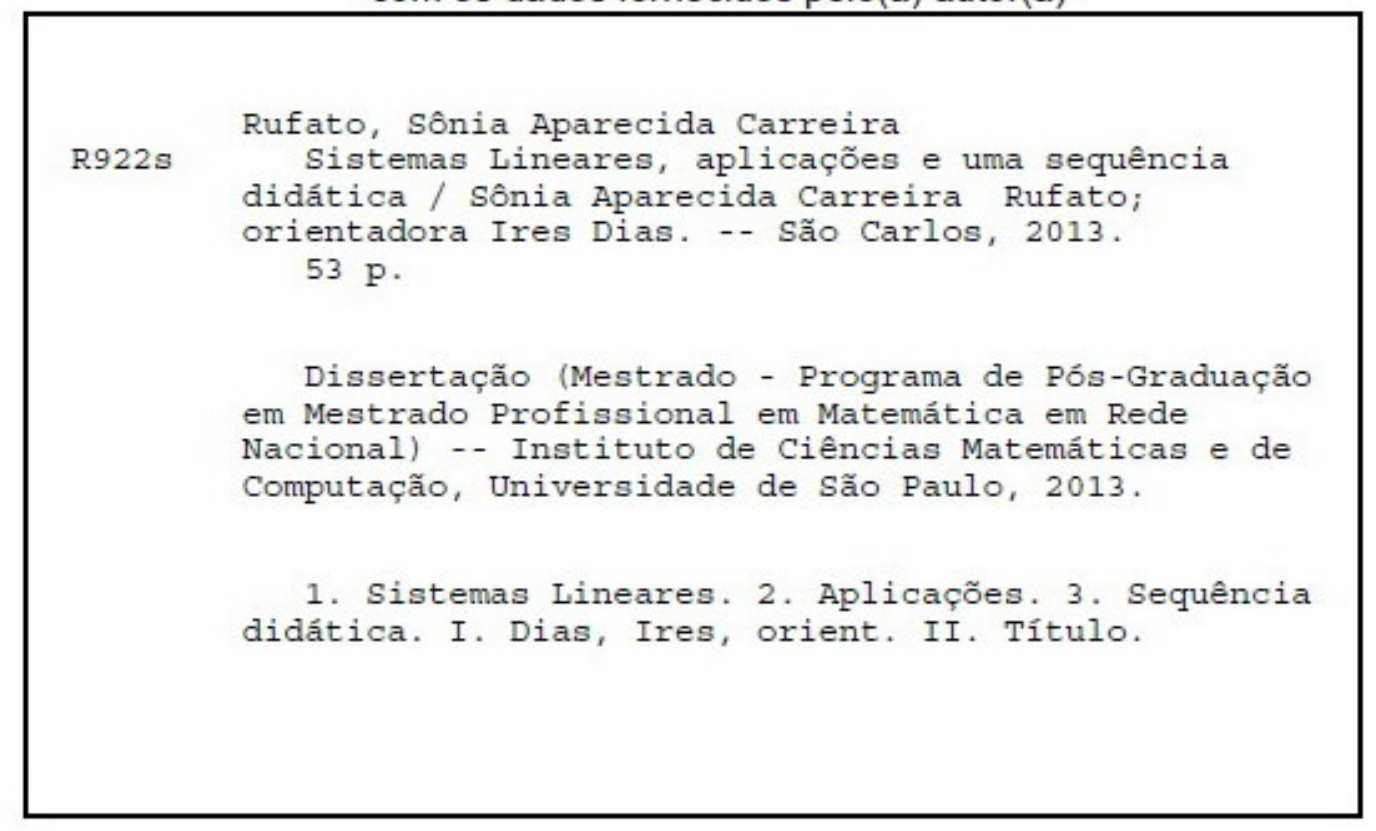




\section{DEDICATÓRIA}

Aos meus filhos Raul Carreira Rufato, Sarah Carreira Rufato e Hugo Carreira Rufato por todo o tempo subtraído de nosso convívio, por toda a força nos momentos críticos.

Ao meu amigo, companheiro e marido Antônio Ailton Rufato por toda sua compreensão, apoio e incentivo nessa grande jornada.

À minha mãe maravilhosa, Maria Tereza Baggio Carreira por toda sua dedicação na coordenação da minha casa para que eu pudesse trilhar novos caminhos... 



\section{AGRADECIMENTOS}

Minha gratidão, em primeiro lugar, a Deus, por estar comigo em todos os momentos $e$ iluminando-me, sendo meu refúgio $e$ fortaleza nos momentos mais difíceis. A ele minha eterna gratidão.

À Professora Doutora, Ires Dias minha "orientadora", que me possibilitou "aprendizagens únicas", por meio do grande incentivo e orientação que me foram concedidos durante essa jornada.

Aos Professores Doutores Hermano de Souza Ribeiro, Luiz Augusto da Costa Ladeira, Miguel Vinícius Santili Frasson, Paulo Leandro Dattori da Silva e Sérgio Luís Zani, por tantos e belos ensinamentos.

Aos Professores Mestres Alex Carlucci Rezende e Nazar Arakelian, pela solicitude em esclarecer todas as dúvidas.

A todos os familiares, pelo suporte $e$ compreensão pelo pouco convívio ao longo desses anos.

Aos colegas de mestrado, por tudo o que com eles aprendi e por partilharem a construção do meu estudo.

A todos os amigos que contribuíram para meu sucesso e para meu crescimento como pessoa. Sou o resultado da confiança e da força de cada um de vocês.

A CAPES, pelo apoio financeiro. 



\section{EPÍGRAFE}

"Educar não é transferir conhecimento, mas criar as possibilidades para a sua própria produção ou a sua construção"

Paulo Freire 



\section{RESUMO}

Este trabalho tem como objetivo salientar a importância do estudo de sistemas de equações lineares no ensino médio através de aplicações que estão inseridas no dia-a-dia, e, tornar significativo o saber matemático, visto que, os alunos demonstram ter uma grande dificuldade em relacionar os conteúdos aprendidos ao longo de suas vidas com situações problemas cotidianas. Para tanto, esse trabalho foi organizado em três capítulos. O capítulo I apresenta uma introdução ao estudo de sistemas de equações lineares sobre números reais, parte da matemática fundamental para a compreensão dos métodos de resolução. O capítulo II apresenta algumas aplicações de sistemas de equações lineares frequentes no dia-a-dia. $\mathrm{O}$ capítulo III apresenta uma proposta de sequência didática de uma situação problema contextualizada para o Ensino Médio.

Palavras-Chave: Sistemas de equações lineares, aplicações de sistemas de equações lineares, aprendizagem significativa, sequência didática. 



\begin{abstract}
The aim in this work is to highlight the importance of studying systems of linear equations for secondary school students through applications that are embedded in day-today, and make significant mathematical knowledge, since students have demonstrated great difficulty relate the content learned throughout their lives with situations everyday problems. For this purpose, this work was organized in three chapters. Chapter I provides an introduction to the study of systems of linear equations over the real numbers, the mathematics fundamental to understanding the methods of resolution. Chapter II presents some applications of linear systems of equations frequently in day-to-day. Chapter III proposes a didactic sequence of a problem situation in context to Secondary School.
\end{abstract}

Keywords: Systems of linear equations, applications of systems of linear equations, meaningful learning, instructional sequence. 



\section{Sumário}

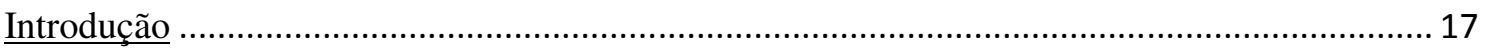

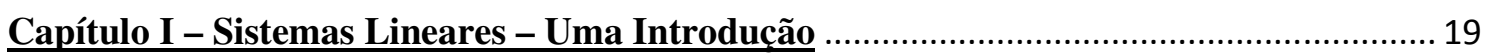

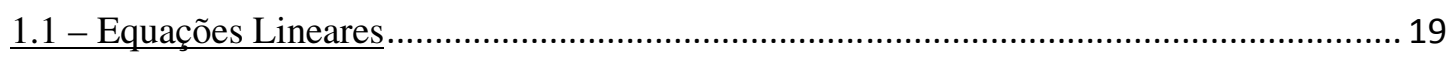

1.2 - Sistemas de Equações Lineares ……………................................................................ 21

1.3 - Resolução de um Sistema de Equações Lineares. ......................................................... 25

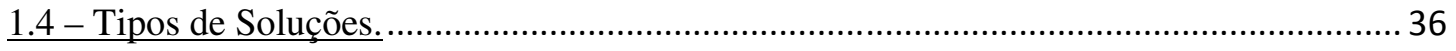

Capítulo II - Aplicacões de Sistemas de Equacões Lineares............................................................. 39

2.1 - Redes Elétricas.................................................................................................................. 39

2.2 - Banceamento de Equações Químicas....................................................................................... 43

2.3 - Controle do fluxo de veículos nas ruas de mão única no horário do rush...........................44

Capítulo III - Sequência Didática - Condicionamento Físico.................................................... 47

3.1 - Descrição, metodologia e aplicação..............................................................................48

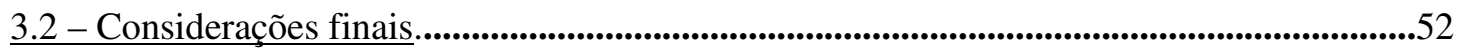

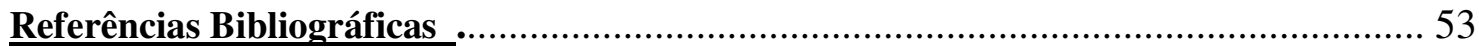







\section{Introdução}

O ensino de matemática desenvolvido nas escolas atualmente, não raramente, tem provocado nos alunos desinteresse quanto ao estudo dos saberes inerentes à ciência matemática. O motivo desse desinteresse pode estar associado a não aplicação dos conteúdos abordados com sua prática do dia a dia, o que acaba por deixar, na maioria das vezes, sem motivação, pois eles não compreendem os significados envolvidos no estudo dos objetos matemáticos.

A não aplicação dos saberes escolar conduz os alunos a um aprendizado mecânico e desprovido de reflexão, pois eles não conseguem relacioná-los com o seu dia a dia. $\mathrm{Na}$ verdade o estudo sem contextualização de um tema pode não permitir a exploração do caráter indagador que ele possui, daí, em muitos casos, não possibilitar a construção significativa do conhecimento. A esse respeito os Parâmetros Curriculares Nacionais (PCN's) afirmam que:

\footnotetext{
... as situações e os desafios que o jovem do ensino médio terá de enfrentar no âmbito escolar, no mundo do trabalho e no exercício da cidadania fazem parte de um processo complexo, no qual as informações são apenas parte de um todo articulado, marcado pela mobilização de conhecimentos e habilidades.

Aprender Matemática de uma forma contextualizada, integrada e relacionada a outros conhecimentos traz em si o desenvolvimento de competências e habilidades que são essencialmente formadoras, à medida que instrumentalizam e estrutura o pensamento do aluno, capacitando-o para compreender e interpretar situações, para se apropriar de linguagens específicas, argumentar, analisar e avaliar, tirar conclusões próprias, tomar decisões, generalizar e para muitas outras ações necessárias à sua formação (p.111).
}

Embora os PCN's recomendem que o ensino de matemática seja desenvolvido relacionando-o com outros conhecimentos, observamos que as temáticas têm sido trabalhadas sem conexão com o cotidiano; é o que se verifica, por exemplo, diante do ensino de Sistemas de Equações Lineares, quando o assunto é geralmente, apresentado de forma desconectada.

A perspectiva de que se estabeleça a conexão dos assuntos abordados e o cotidiano vai ao encontro de objetivos traçados nos PCN's a fim de melhorar o ensino de matemática, dos quais destacamos:

- Compreender a Matemática como ciência autônoma, que investiga relações, formas e eventos e desenvolve maneiras próprias de descrever e interpretar o mundo. 
- Adquirir uma compreensão do mundo da qual a Matemática é parte integrante, através dos problemas que ela consegue resolver e dos fenômenos que podem ser descritos por meio de seus modelos e representações.

- Reconhecer relações entre a Matemática e outras áreas do conhecimento, percebendo sua presença nos mais variados campos de estudo e da vida humana, seja nas demais ciências, como a Física, Química e Biologia, seja nas ciências humanas e sociais, como a Geografia ou a Economia, ou ainda nos mais diversos setores da sociedade, como na agricultura, na saúde, nos transportes e na moradia.

Os objetivos apresentados demonstram as recomendações dos próprios PCN's para que se ensine matemática fazendo uso de conexões entre os saberes escolares e a compreensão de mundo, no entanto, ainda encontramos situações de ensino desenvolvidas mecanicamente, relegando aos alunos a difícil tarefa de montar verdadeiros "quebra cabeças", esperando que consigam estabelecer conexões entre as várias peças apresentadas.

Neste trabalho nos propomos a apresentar uma sequência didática, que pode ser aplicada a alunos de uma segunda série do ensino médio, visando ajudar os alunos a compreender que o assunto sistemas lineares tem aplicabilidade no dia a dia.

Para tanto, no capítulo I, apresentamos uma introdução ao estudo de Sistemas Lineares necessária para facilitar a compreensão do restante do trabalho para o professor e ou aluno que pretenda utilizá-lo.

No capítulo II, apresentamos três aplicações de Sistemas Lineares com a sugestão de que em série do ensino médio elas podem ser introduzidas.

Finalmente, no capítulo III, apresentamos a descrição da sequência didática, juntamente com a metodologia utilizada e considerações finais. 


\section{Capítulo I}

\section{Sistemas Lineares - Uma Introdução}

Neste capítulo apresentaremos uma introdução ao estudo de sistemas de equações lineares sobre os números reais, contendo definições, exemplos e técnicas de resolução. Tal estudo servirá de suporte teórico para a compreensão do restante do trabalho e, contêm somente os resultados necessários para a compreensão do mesmo.

\section{1 - Equações Lineares}

Lembremos que a equação geral de uma reta no plano, $\mathbb{R}^{2}$, é da forma $a x+b y=c$, e a equação geral de um plano em $\mathbb{R}^{3}$ é da forma $a x+b y+c z=d$, onde, $a, b, c$ e $d$ são números reais não todos nulos dados. Equações como estas são chamadas equações lineares.

\subsection{1 - Definição}

Uma equação linear nas $n$ variáveis $x_{1}, x_{2}, \ldots, x_{n}$ é uma equação que pode ser escrita na forma $a_{1} x_{1}+a_{2} x_{2}+\cdots+a_{n} x_{n}=b$, onde os coeficientes $a_{1}, a_{2}, \ldots, a_{n}$ e o termo independente $b$ são números reais dados.

No que segue, a menos de menção contrária, todos os coeficientes e termos constantes das equações lineares citadas, serão números reais.

\subsection{2- Exemplos:}

(a) As seguintes equações são lineares: 


$$
\begin{aligned}
& 3 x-4 y=-1 ; \\
& r-\frac{1}{2} s+2 t=\frac{1}{3} ; \\
& x_{1}+4 x_{2}=5+5 x_{3}+x_{4} ; \\
& \sqrt{2} x+\frac{\pi}{4} y-\operatorname{sen}\left(\frac{\pi}{5}\right) z=1 ; \\
& 3,2 x_{1}-0,01 x_{2}=4,6 .
\end{aligned}
$$

(b) As seguintes equações não são lineares:

$$
\begin{aligned}
& x y+3 z=1 ; \\
& x_{1}^{2}-2 x_{2}^{3}+x_{3}=5 ; \\
& \frac{x}{y}+z=2 \\
& \sqrt{2 x}+\frac{\pi}{4} y-\operatorname{sen}\left(\frac{\pi z}{5}\right)=1 ; \\
& \operatorname{sen} x_{1}-3 x_{2}+2^{x_{3}}=0 .
\end{aligned}
$$

Equações lineares não contêm produtos, recíprocas, radicais, ou outras funções não lineares nas variáveis. As variáveis aparecem somente na potência 1 e multiplicadas por constantes (números reais).

\subsection{3 - Definição}

Uma solução de uma equação linear $a_{1} x_{1}+a_{2} x_{2}+\cdots+a_{n} x_{n}=b$ é uma $n$-upla de números reais $\left(k_{1}, k_{2}, \ldots, k_{n}\right)$ cujas coordenadas satisfazem à equação, ou seja, a igualdade $a_{1} k_{1}+a_{2} k_{2}+\cdots+a_{n} k_{n}=b$ é verdadeira.

\subsection{4 - Exemplo}

Considere a equação linear $3 x_{1}+4 x_{2}-5 x_{3}-x_{4}=5$. A sequência $(1,-2,-2,0)$ é solução da equação, pois, $3.1+4 .(-2)-5 .(-2)-1.0=5$ é verdadeira. Já $(1,1,2,1)$ não é solução, pois $3.1+4.1-5.2-1.1=5$ não é uma sentença verdadeira.

Note que da equação obtemos $x_{1}=\frac{5-4 x_{2}+5 x_{3}+x_{4}}{3}$. Assim, escolhendo valores para as variáveis $x_{2}, x_{3}$ e $x_{4}$ obtemos um valor para $x_{1}$ e, consequentemente uma nova solução para a equação dada. 
Considere agora a equação linear $0 x_{1}+0 x_{2}+0 x_{3}=4$. Todos os coeficientes são zero e o termo independente é diferente de zero. Claramente esta equação não admite solução, ou seja, não existe uma sequência de números reais $\left(k_{1}, k_{2}, k_{3}\right)$ satisfazendo a equação.

\section{2 - Sistemas de Equações Lineares}

Muitos problemas são modelados matematicamente por sistemas de equações lineares em diversas áreas do conhecimento, desde a antiguidade. Por volta de 1.800 a.C já eram considerados pelos babilônios os sistemas com duas equações lineares e resolvidos por um método que chamamos hoje de método de eliminação gaussiana ${ }^{1}$.

${ }^{1}$ Em homenagem a Carl Friedrich Gauss (Alemanha, 1777-1855), considerado um dos maiores matemáticos de todos os tempos.

\subsection{1 - Definição}

Um sistema de equações lineares é um conjunto finito de equações lineares, cada uma delas com as mesmas variáveis, ou seja, um conjunto de $m$ equações lineares com $n$ variáveis da forma

$$
S:\left\{\begin{array}{c}
a_{11} x_{1}+a_{12} x_{2}+\cdots+a_{1 n} x_{n}=b_{1} \\
a_{21} x_{1}+a_{22} x_{2}+\cdots+a_{2 n} x_{n}=b_{2} \\
\cdots \quad \cdots \quad \cdots \quad \cdots \quad \cdots \quad \cdots \quad \cdots \quad \cdots \\
a_{m 1} x_{1}+a_{m 2} x_{2}+\cdots+a_{m n} x_{n}=b_{m}
\end{array}\right.
$$

onde $a_{i j}, b_{i} \in \mathbb{R}$, para $1 \leq i \leq m$ e $1 \leq j \leq n$.

Uma solução do sistema $\mathrm{S}$ é uma $n$-upla de números reais $u=\left(k_{1}, k_{2}, \ldots, k_{n}\right)$ que é solução de cada uma das $m$ equações do sistema. O conjunto solução do sistema $\mathrm{S}$ é o conjunto formado por todas as soluções de $\mathrm{S}$.

No que segue a expressão "resolver o sistema" será usada significando encontrar o conjunto solução do sistema de equações lineares dado.

Os próximos três exemplos ilustram os possíveis conjuntos soluções de um sistema de equações lineares, com coeficientes reais o que, na realidade, classifica os tipos de sistemas lineares possíveis. 


\subsection{2 - Exemplo}

Resolva o seguinte sistema linear:

$$
S_{1}:\left\{\begin{array}{c}
x+y=4 \\
2 x-y=2
\end{array}\right.
$$

Somando as duas equações obtemos $3 x=6$, o que resulta em $x=2$. Substituindo $x=2$ na primeira equação, obtemos $y=2$. Note que $u=(2,2)$ também é solução da segunda equação, ou seja, $u=(2,2)$ é uma solução de $S_{1}$.

Usando que cada equação de $S_{1}$ é uma equação de uma reta em $\mathbb{R}^{2}$, obtemos que $u=(2,2)$ é a única solução de $S_{1}$, ou seja, o conjunto solução de $S_{1}$ tem um único elemento. Veja abaixo o gráfico das equações de $S_{1}$.

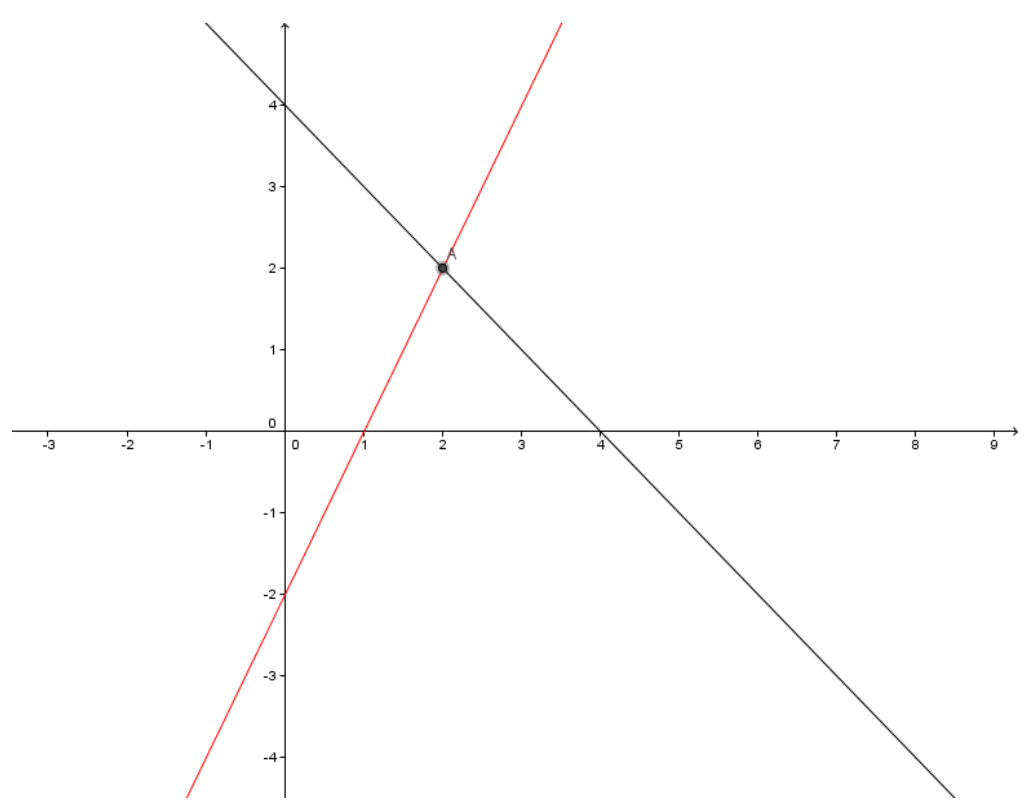

Figura 1: Gráfico das equações de $S_{1}$

\subsection{3 - Exemplo}

Resolva o seguinte sistema de equações lineares: 


$$
S_{2}:\left\{\begin{array}{l}
x+y=2 \\
2 x+2 y=4
\end{array}\right.
$$

A segunda equação de $S_{2}$ é exatamente o dobro da primeira, assim, as soluções de $S_{2}$ são as soluções da primeira equação, ou seja, os pontos da reta $y=2-x$. Consequentemente o conjunto solução de $S_{2}$ é $\{(k, 2-k) ; k \in \mathbb{R}\}$ que é um conjunto infinito. Veja o gráfico abaixo:

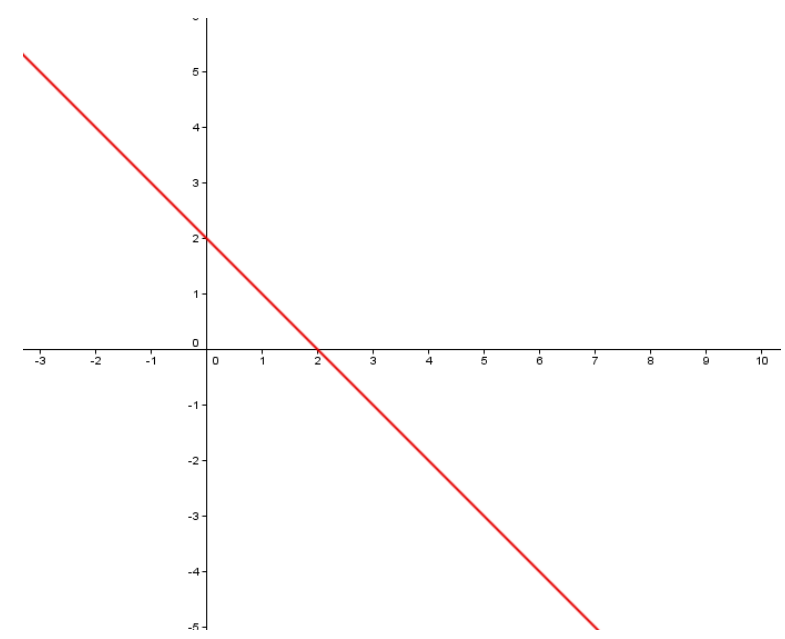

Figura 2: Gráfico das equações de $S_{2}$

\subsection{4 - Exemplo}

Resolva o seguinte sistema de equações lineares:

$$
S_{3}:\left\{\begin{array}{l}
x+y=4 \\
x+y=3
\end{array}\right.
$$

Não existem números reais $x$ e $y$ cuja soma seja 4 e 3 simultaneamente, ou seja, $S_{3}$ não admite solução.

Geometricamente podemos ver que as equações lineares que compõe $S_{3}$ são retas paralelas e, portanto, este é um sistema cujo conjunto solução é o conjunto vazio. 


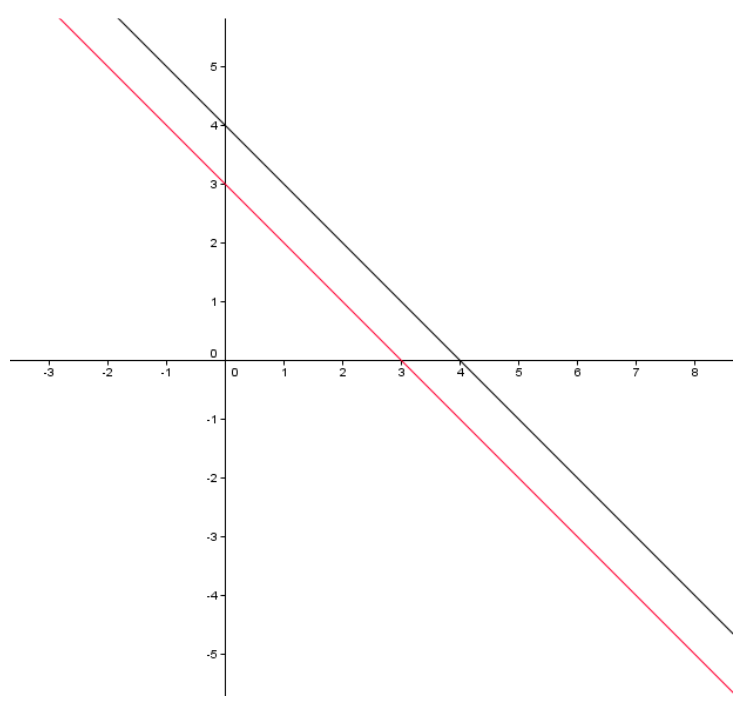

Figura 3: Gráfico das equações de $S_{3}$

Baseado nos exemplos acima definimos:

\subsection{5 - Definição}

Um sistema de equações lineares é dito ser possível, (ou compatível, ou ainda consistente) quando tem pelo menos uma solução, isto é, seu conjunto solução é diferente do conjunto vazio.

Um sistema possível é dito ser determinado quando admite uma única solução e indeterminado quando admite mais de uma solução.

Dizemos que um sistema de equações lineares que não admite soluções é um sistema impossível, (ou incompatível, ou ainda inconsistente).

Mostraremos mais adiante que a classificação completa dos sistemas de equações lineares, em termos do número de soluções é:

Para um sistema de equações lineares $\mathrm{S}$ com coeficientes reais, temos uma e somente uma das possibilidades abaixo verdadeiras:

(a) S admite uma única solução, ou seja, é possível e determinado.

(b) S admite infinitas soluções, ou seja, é possível e indeterminado.

(c) S não admite solução, ou seja, é impossível. 


\section{3 - Resolução de um Sistema de Equações Lineares.}

Para a resolução de um sistema de equações lineares sobre os números reais, apresentaremos um procedimento geral e sistemático baseados na ideia de reduzir o sistema linear dado, ou equivalentemente, sua matriz associada, de forma a obter um sistema e ou matriz mais simples que tenha o mesmo conjunto solução.

\subsection{1 - Definição}

Dizemos que dois sistemas de equações lineares são equivalentes quando eles têm o mesmo conjunto solução.

\subsection{2 - Proposição}

As operações elementares abaixo, quando aplicada em um sistema de equações lineares, da origem a sistemas equivalentes.

(I) Permutação de duas equações;

(II) Multiplicação de uma equação por um número real diferente de zero;

(III) Substituição de uma equação por sua soma com outra equação previamente multiplicada por um número real diferente de zero.

Demonstração: Considere o sistema linear

$$
S:\left\{\begin{array}{c}
a_{11} x_{1}+a_{12} x_{2}+\cdots+a_{1 n} x_{n}=b_{1} \\
a_{21} x_{1}+a_{22} x_{2}+\cdots+a_{2 n} x_{n}=b_{2} \\
\cdots \quad \cdots \quad \cdots \quad \cdots \\
a_{m 1} x_{1}+a_{m 2} x_{2}+\cdots+a_{m n} x_{n}=b_{m}
\end{array}\right.
$$

Claramente as operações (I) e (II) geram sistemas equivalentes.

Para mostrarmos que aplicando a operação (III) em S, obtemos um sistema equivalente, sem perda de generalidade podemos supor que trocamos a primeira equação de $S$ pela soma desta com a segunda equação multiplicada por $\alpha \in \mathbb{R} \operatorname{com} \alpha \neq 0$. Obtemos então o sistema $S^{\prime}$ : 


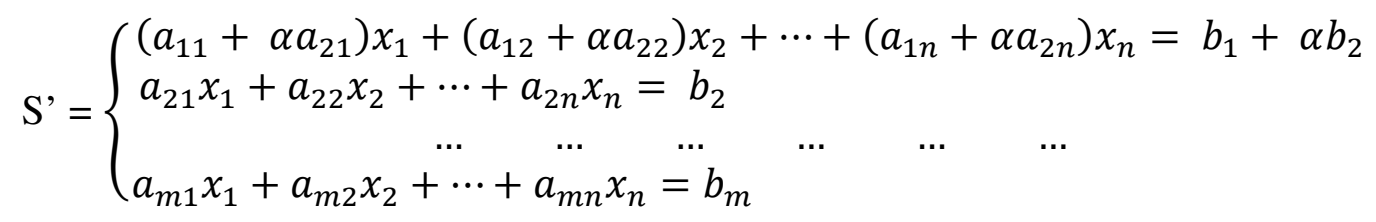

Queremos mostrar que S e S' são sistemas equivalentes. Dado $u=\left(k_{1}, \ldots, k_{n}\right)$ uma $n$ - upla de números reais, temos que $u$ é solução de $\mathrm{S}$ se, e somente se

$$
\left\{\begin{array}{c}
a_{11} k_{1}+a_{12} k_{2}+\cdots+a_{1 n} k_{n}=b_{1} \\
a_{21} k_{1}+a_{22} k_{2}+\cdots+a_{2 n} k_{n}=b_{2} \\
\cdots \quad \cdots \quad \cdots \quad \cdots \\
a_{m 1} k_{1}+a_{m 2} k_{2}+\cdots+a_{m n} k_{n}=b_{m}
\end{array}\right.
$$

são igualdades de números reais.

Como $\alpha \neq 0$, multiplicando a segunda igualdade por $\alpha$ (respectivamente por $\alpha^{-1}$ ), ou seja, aplicando a operação (II), obtemos que as igualdades acima são verdadeiras se, e somente se o são as abaixo:

$$
\left\{\begin{array}{l}
a_{11} k_{1}+a_{12} k_{2}+\cdots+a_{1 n} k_{n}=b_{1} \\
\alpha a_{21} k_{1}+\alpha a_{22} k_{2}+\cdots+\alpha a_{2 n} k_{n}=\alpha b_{2} \\
\cdots \quad \cdots \quad \cdots \quad \cdots \\
a_{m 1} k_{1}+a_{m 2} k_{2}+\cdots+a_{m n} k_{n}=b_{m}
\end{array}\right.
$$

Trocando a primeira igualdade pela soma (respectivamente pela diferença) das duas primeiras igualdades, obtemos que as igualdades acima são verdadeiras se, e somente se

$$
\left\{\begin{array}{l}
\left(a_{11}+\alpha a_{21}\right) k_{1}+\left(a_{12}+\alpha a_{22}\right) k_{2}+\cdots+\left(a_{1 n}+\alpha a_{2 n}\right) k_{n}=b_{1}+\alpha b_{2} \\
\alpha a_{21} k_{1}+\alpha a_{22} k_{2}+\cdots+\alpha a_{2 n} k_{n}=\alpha b_{2} \\
\cdots \quad \cdots \quad \cdots \quad \cdots \quad \cdots \\
a_{m 1} k_{1}+a_{m 2} k_{2}+\cdots+a_{m n} k_{n}=b_{m}
\end{array}\right.
$$

Aplicando novamente a operação (II), obtemos que $u=\left(k_{1}, \ldots, k_{n}\right)$ satisfaz as igualdades acima se, e somente se é uma solução de $S$, o que mostra que $S$ e $S$ ' são equivalentes.

Observe que ao aplicarmos as operações elementares a um sistema linear, estamos operando somente com os coeficientes do sistema e não com as variáveis. Assim, para economia de escrita, registraremos de maneira apropriada apenas os coeficientes e os termos constantes do sistema em uma matriz, chamada de matriz completa ou matriz aumentada do sistema, ou seja: 


\subsection{3 - Definição}

Dado o sistema linear

$$
S:\left\{\begin{array}{c}
a_{11} x_{1}+a_{12} x_{2}+\cdots+a_{1 n} x_{n}=b_{1} \\
a_{21} x_{1}+a_{22} x_{2}+\cdots+a_{2 n} x_{n}=b_{2} \\
\cdots \quad \cdots \quad \cdots \quad \cdots \\
\cdots \quad x_{m 1} x_{1}+a_{m 2} x_{2}+\cdots+a_{m n} x_{n}=b_{m}
\end{array}\right.
$$

A matriz aumentada de S é a matriz

$$
\left[\begin{array}{cccc|c}
a_{11} & a_{12} & \cdots & a_{1 n} & b_{1} \\
a_{21} & a_{22} & \cdots & a_{2 n} & b_{2} \\
\vdots & \vdots & \cdots & \vdots & \vdots \\
a_{m 1} & a_{m 2} & \cdots & a_{m n} & b_{m}
\end{array}\right]
$$

Onde as $n$ primeiras colunas contêm os coeficientes das variáveis na ordem certa, a última coluna contêm os termos constantes e a barra vertical serve para nos lembrar do sinal de igual nas equações.

No próximo exemplo veremos como resolver um sistema de equações lineares aplicando as operações elementares no sistema e paralelamente operações análogas nas linhas da matriz aumentada.

\subsection{4- Exemplo}

Resolva o seguinte sistema de equações lineares:

$$
S:\left\{\begin{array}{l}
x-y-z=2 \\
3 x-3 y+2 z=16 \\
2 x-y+z=9
\end{array}\right.
$$

A matriz aumentada associada a $S$ é $A=\left[\begin{array}{rrr|r}1 & -1 & -1 & 2 \\ 3 & -3 & 2 & 16 \\ 2 & -1 & 1 & 9\end{array}\right]$ 
Subtraindo três vezes a primeira equação da segunda, obtemos:

$$
\left\{\begin{array}{l}
x-y-z=2 \\
5 z=10 \\
2 x-y+z=9
\end{array}\right.
$$

Subtraindo duas vezes a primeira equação da terceira, obtemos:

$\left\{\begin{array}{l}x-y-z=2 \\ 5 z=10 \\ y+3 z=5\end{array}\right.$

Trocando as equações 2 e 3 obtemos:
Subtraindo três vezes a primeira linha da segunda, obtemos:

$$
\left[\begin{array}{rrr|r}
1 & -1 & -1 & 2 \\
0 & 0 & 5 & 10 \\
2 & -1 & 1 & 9
\end{array}\right]
$$

Subtraindo duas vezes a primeira linha da terceira, obtemos:

$$
\left[\begin{array}{rrr|r}
1 & -1 & -1 & 2 \\
0 & 0 & 5 & 10 \\
0 & 1 & 3 & 5
\end{array}\right]
$$

Trocando as linhas 2 e 3 obtemos:

$$
\left[\begin{array}{rrr|r}
1 & -1 & -1 & 2 \\
0 & 1 & 3 & 5 \\
0 & 0 & 5 & 10
\end{array}\right]
$$

Obtemos então que S é equivalente ao sistema

$$
S^{\prime}=\left\{\begin{array}{l}
x-y-z=2 \\
y+3 z=5 \\
5 z=10
\end{array}\right.
$$

Resolvendo S' por substituição de trás para a frente, temos da última equação que $z=2$, substituindo na segunda obtemos $y=5-3.2=-1$. Da primeira equação obtemos $x=2+(-1)+(2)=3$, ou seja, $u=(3,-1,2)$ é a única solução de $\mathrm{S}^{\prime}$ e, consequentemente a única solução do sistema dado $S$.

Notemos que a matriz aumentada associada à S' é uma matriz triangular. Nem sempre será possível reduzir a matriz de um sistema à forma triangular, mas sempre podemos conseguir um formato de escada nos elementos não nulos da matriz final, que será a forma escalonada por linhas da matriz original.

A palavra escalonar vem da palavra latina scaba, que significa "escada" ou "degrau". Escalonar uma matriz significa dar a ela a forma de escada. 


\subsection{5 - Definição}

Uma matriz está na forma escalonada por linhas quando satisfaz as seguintes propriedades:

(I) Todas as linhas que consistem inteiramente de zeros estão na parte inferior da matriz.

(II) Em cada linha não nula, i. é, que não consiste só de zeros, o primeiro elemento não nulo, chamado de elemento líder, está em uma coluna à esquerda de qualquer outro elemento líder abaixo dele.

Conforme observado no Exemplo 1.3.4, aplicando as operações elementares no sistema linear, obtemos operações correspondentes na matriz aumentada do sistema, as quais chamaremos de operações elementares com as linhas.

\subsection{6 - Definição}

Dada uma matriz A, as seguintes operações sobre A, são ditas serem operações elementares com as linhas de A:

(I) Trocar as posições de duas linhas de A.

(II) Multiplicar uma linha de A por um número real (um escalar) não nulo.

(III) Trocar uma linha de A pela soma dela com um múltiplo escalar de outra linha.

Usaremos a seguinte notação para as três operações elementares com linhas:

(I) $\quad L_{i} \leftrightarrow L_{j}$, significa trocar as linhas $i$ e $j$.

(II) $\quad \alpha . L_{i}$, significa multiplicar a linha $i$ pelo número real $\alpha$.

(III) $\quad L_{i}+\alpha L_{j}$, significa trocar a linha $i$ pela soma dela $\operatorname{com} \alpha$ vezes a linha $j$.

\subsection{7- Exemplo}

Reduza a seguinte matriz à forma escalonada: 


$$
\left[\begin{array}{rrrrr}
1 & 2 & -4 & -4 & 5 \\
2 & 4 & 0 & 0 & 2 \\
2 & 3 & 2 & 1 & 5 \\
-1 & 1 & 3 & 6 & 5
\end{array}\right]
$$

Trabalhamos coluna por coluna, da esquerda para a direita e de cima para baixo. A estratégia é criar um elemento líder em uma coluna e usá-lo para criar zeros sob ele. O elemento escolhido para ser o elemento líder é chamado pivô. Embora não seja estritamente necessário, às vezes é conveniente usar a segunda operação elementar para transformar o elemento líder em 1.

Começamos por anular os elementos da primeira coluna, abaixo do líder 1 na primeira linha:

$$
\left[\begin{array}{rrrrr}
1 & 2 & -4 & -4 & 5 \\
2 & 4 & 0 & 0 & 2 \\
2 & 3 & 2 & 1 & 5 \\
-1 & 1 & 3 & 6 & 5
\end{array}\right] \stackrel{\substack{L_{2}-2 L_{1}-2 L_{1} \\
L_{4}+L_{1}}}{\longrightarrow}\left[\begin{array}{rrrrr}
1 & 2 & -4 & -4 & 5 \\
0 & 0 & 8 & 8 & -8 \\
0 & -1 & 10 & 9 & -5 \\
0 & 3 & -1 & 2 & 10
\end{array}\right]
$$

A primeira coluna agora está como queremos; o próximo passo é obter um elemento líder na segunda linha, com o objetivo de chegar à forma escalonada. Neste caso, fazemos isso trocando as linhas. (Poderíamos também somar a linha 3 ou 4 à linha 2.)

$$
\left[\begin{array}{rrrrr}
1 & 2 & -4 & -4 & 5 \\
0 & 0 & 8 & 8 & -8 \\
0 & -1 & 10 & 9 & -5 \\
0 & 3 & -1 & 2 & 10
\end{array}\right] \stackrel{L_{2} \leftrightarrow L_{3}}{\longrightarrow}\left[\begin{array}{rrrrr}
1 & 2 & -4 & -4 & 5 \\
0 & -1 & 10 & 9 & -5 \\
0 & 0 & 8 & 8 & -8 \\
0 & 3 & -1 & 2 & 10
\end{array}\right]
$$

Desta vez o pivô é -1. Obtemos zeros no restante da segunda coluna usando o elemento líder -1 na linha 2:

$$
\left[\begin{array}{rrrrr}
1 & 2 & -4 & -4 & 5 \\
0 & -1 & 10 & 9 & -5 \\
0 & 0 & 8 & 8 & -8 \\
0 & 3 & -1 & 2 & 10
\end{array}\right] \stackrel{L_{4}+3 L_{2}}{\longrightarrow}\left[\begin{array}{rrrrr}
1 & 2 & -4 & -4 & 5 \\
0 & -1 & 10 & 9 & -5 \\
0 & 0 & 8 & 8 & -8 \\
0 & 0 & 29 & 29 & -5
\end{array}\right]
$$

A coluna 2 agora está na forma desejada. Note que já temos um elemento líder na coluna 3, nós o escolhemos como pivô e começamos a tarefa de obter zeros sob ele. Isso ficará mais fácil se primeiramente dividirmos a linha 3 por 8 : 


$$
\left[\begin{array}{rrrrr}
1 & 2 & -4 & -4 & 5 \\
0 & -1 & 10 & 9 & -5 \\
0 & 0 & 8 & 8 & -8 \\
0 & 0 & 29 & 29 & -5
\end{array}\right] \stackrel{\frac{1}{8} L_{3}}{\rightarrow}\left[\begin{array}{rrrrr}
1 & 2 & -4 & -4 & 5 \\
0 & -1 & 10 & 9 & -5 \\
0 & 0 & 1 & 1 & -1 \\
0 & 0 & 29 & 29 & -5
\end{array}\right]
$$

Agora usando o elemento líder 1 da coluna 3 obtemos:

$$
\left[\begin{array}{rrrrr}
1 & 2 & -4 & -4 & 5 \\
0 & -1 & 10 & 9 & -5 \\
0 & 0 & 1 & 1 & -1 \\
0 & 0 & 29 & 29 & -5
\end{array}\right] \stackrel{L_{4}-29 L_{3}}{\longrightarrow}\left[\begin{array}{rrrrr}
1 & 2 & -4 & -4 & 5 \\
0 & -1 & 10 & 9 & -5 \\
0 & 0 & 1 & 1 & -1 \\
0 & 0 & 0 & 0 & 24
\end{array}\right]
$$

Com esse passo, reduzimos a matriz dada à forma escalonada.

\subsection{8- Observações:}

(I) A forma escalonada da matriz não é única.

(II) O elemento líder de cada linha é usado para obter zeros abaixo dele.

(III) Os pivôs não são necessariamente os elementos que estavam originalmente nas posições ocupadas pelos elementos líderes. No exemplo anterior, os pivôs eram $1,-1,8$, e 24 e a matriz original tinha $1,4,2$, e 5 nessas posições.

(IV) Uma vez que tenhamos anulado os elementos sob o elemento líder em uma coluna, aquela coluna não muda mais. Em outras palavras, a forma escalonada por linhas é construída da esquerda para a direita e de cima para baixo.

Como no caso de operações elementares aplicadas em sistemas lineares, operações elementares com linhas de matrizes são reversíveis, ou seja, podem ser desfeitas. Logo, se uma operação elementar sobre as linhas transforma a matriz A na matriz B, então existe outra operação elementar sobre as linhas de B que a transforma na matriz A.

Analogamente ao caso dos sistemas, definimos: 


\subsection{9 - Definição}

As matrizes A e B são linha-equivalentes se existir uma sequência finita de operações elementares com as linhas que converte A em B.

As matrizes

$$
\left[\begin{array}{rrrrr}
1 & 2 & -4 & -4 & 5 \\
2 & 4 & 0 & 0 & 2 \\
2 & 3 & 2 & 1 & 5 \\
-1 & 1 & 3 & 6 & 5
\end{array}\right] \quad e \quad\left[\begin{array}{rrrrr}
1 & 2 & -4 & -4 & 5 \\
0 & -1 & 10 & 9 & -5 \\
0 & 0 & 1 & 1 & -1 \\
0 & 0 & 0 & 0 & 24
\end{array}\right]
$$

do Exemplo 1.3.7, são linha- equivalentes.

O próximo resultado é imediato.

\subsubsection{0-Teorema}

Dois sistemas lineares são equivalentes se, e somente se suas correspondentes matrizes aumentadas são linhas-equivalentes.

Em geral não é imediato ver que duas matrizes são linha-equivalentes, o próximo resultado auxilia nesta tarefa.

\subsubsection{1 - Teorema}

Duas matrizes são linhas-equivalentes se, e somente se ambas podem ser reduzidas à mesma forma escalonada por linhas.

Demonstração: Sejam A e B duas matrizes. Se A e B são linha-equivalentes, então é imediato que, utilizando operações elementares por linhas ambas podem ser convertidas à mesma matriz escalonada por linhas.

Reciprocamente, se A e B tem a mesma forma escalonada por linhas C, então existem finitas operações elementares por linhas que convertem A em C e B em C.

Aplicando em A as operações que a convertem em $\mathrm{C}$ e depois as operações elementares reversas que convertem $\mathrm{B}$ em $\mathrm{C}$, obtemos um numero finito de operações elementares por linhas que convertem A em B, ou seja, A e B são linhas-equivalentes. 
Quando uma redução por linhas é aplicada à matriz aumentada de um sistema de equações lineares, obtemos um sistema equivalente que pode ser resolvido facilmente por substituição de trás para a frente, como no Exemplo 1.3.4. Tal processo é conhecido como método de eliminação de Gauss, ou método de eliminação gaussiana. Tal método era conhecido pelos chineses no século 3 a.C., mas recebe o nome de Gauss por sua redescoberta em um artigo no qual ele resolve um sistema de equações lineares para descrever a órbita de um asteróide.

\subsubsection{2 - O Método de Eliminação de Gauss}

Dado um sistema de equações lineares S, o método de Eliminação de Gauss consiste em seguir os seguintes passos:

(I) Escreva a matriz aumentada do sistema.

(II) Use operações elementares com as linhas para reduzir a matriz aumentada à forma escalonada por linhas, i.é, escalone a matriz aumentada.

(III) Usando substituição de trás para a frente, resolva o sistema correspondente à matriz linha- reduzida de (II).

O conjunto solução encontrado em (III) é o conjunto solução do sistema de equações lineares $\mathrm{S}$ dado.

\subsubsection{3 - Exemplo}

Resolva o sistema

$$
S:\left\{\begin{array}{l}
x_{1}+x_{2}+x_{3}=3 \\
2 x_{1}+3 x_{2}+x_{3}=5 \\
x_{1}-x_{2}-2 x_{3}=-5
\end{array}\right.
$$

A matriz aumentada do sistema $\mathrm{S}$ é

$$
\left[\begin{array}{rrr|r}
1 & 1 & 1 & 3 \\
2 & 3 & 1 & 5 \\
1 & -1 & -2 & -5
\end{array}\right]
$$

Continuamos o processo reduzindo essa matriz à forma escalonada por linhas: 


$$
\left[\begin{array}{rrr|r}
1 & 1 & 1 & 3 \\
2 & 3 & 1 & 5 \\
1 & -1 & -2 & -5
\end{array}\right] \stackrel{L_{2}-2 L_{1}}{\longrightarrow}\left[\begin{array}{rrr|r}
1 & 1 & 1 & 3 \\
0 & 1 & -1 & -1 \\
0 & -2 & -3 & -8
\end{array}\right] \stackrel{L_{3}+2 L_{2}}{\longrightarrow}\left[\begin{array}{rrr|r}
1 & 1 & 1 & 3 \\
0 & 1 & -1 & -1 \\
0 & 0 & -5 & -10
\end{array}\right]
$$

O sistema correspondente agora é:

$$
\left\{\begin{array}{l}
x_{1}+x_{2}+x_{3}=3 \\
x_{2}-x_{3}=-1 \\
-5 x_{3}=-10
\end{array}\right.
$$

A substituição de trás para frente nos dá $x_{3}=2, x_{2}=1, x_{1}=0$, e, portanto, a única solução de $S$ é $(0,1,2)$.

Uma modificação do método de eliminação de Gauss simplifica bastante a resolução do sistema reduzido e é particularmente útil quando os cálculos estão sendo feitos à mão em um sistema com infinitas soluções. Essa variante é conhecida como método de eliminação de Gauss-Jordan, devido à contribuição de Wilhelm Jordan, professor alemão de geodésia, que criou um método sistemático de substituição de trás para a frente estreitamente relacionado com o método que hoje leva o seu nome. Tal método baseia-se em reduzir ainda mais a matriz aumentada do sistema.

\subsubsection{4- Definição}

Uma matriz está na forma escalonada reduzida (por linhas) se ela satisfaz as seguintes propriedades:

(I) Quaisquer linhas que consistem inteiramente de zeros estão na parte inferior da matriz.

(II) O elemento líder em cada linha não nula é igual a 1 ( chamado 1 líder).

(III) Cada coluna que contém 1 líder tem zeros em todas as outras posições.

A matriz abaixo está na forma escalonada reduzida.

$$
\left[\begin{array}{rrrrrrr}
1 & 2 & 0 & 0 & -3 & 1 & 0 \\
0 & 0 & 1 & 0 & 4 & -1 & 0 \\
0 & 0 & 0 & 1 & 3 & 2 & 0 \\
0 & 0 & 0 & 0 & 0 & 0 & 1 \\
0 & 0 & 0 & 0 & 0 & 0 & 0
\end{array}\right]
$$


É claro que, depois de uma matriz ter sido escalonada, mais operações elementares com as linhas, irão leva-la à forma escalonada reduzida. O que não é imediato é que a forma escalonada reduzida de uma matriz é única, para ver uma demonstração deste fato, veja o artigo "The reduced row echelon form of a matrix is unique: A simple proof", de Thomas Yuster, Mathematics Magazine, V.57, n² (Março, 1984), p.93-94.

\subsubsection{5- O método de eliminação de Gauss-Jordan}

Dado um sistema de equações lineares S, o método de eliminação de Gauss-Jordan consiste em:

(I) Escrever a matriz aumentada do sistema.

(II) Usar operações elementares com linhas para reduzir a matriz aumentada à forma escalonada reduzida.

(III) Se o sistema resultante for possível, resolvê-lo.

O conjunto solução obtido em (III) é o conjunto solução do sistema S.

Como observado anteriormente, este método é útil quando o conjunto solução do sistema é infinito.

\subsubsection{6 - Exemplo}

Resolva o sistema de equações lineares

$$
S:\left\{\begin{array}{l}
w-x-y+2 z=1 \\
2 w-2 x-y+3 z=3 \\
-w+x-y=-3
\end{array}\right.
$$

A matriz aumentada de S é

$$
\left[\begin{array}{rrrr|r}
1 & -1 & -1 & 2 & 1 \\
2 & -2 & -1 & 3 & 3 \\
-1 & 1 & -1 & 0 & -3
\end{array}\right]
$$

Que pode ser escalonada como segue: 


$$
\left[\begin{array}{rrrr|r}
1 & -1 & -1 & 2 & 1 \\
2 & -2 & -1 & 3 & 3 \\
-1 & 1 & -1 & 0 & -3
\end{array}\right] \stackrel{L_{2}-2 L_{1}}{\longrightarrow}\left[\begin{array}{rrrr|r}
1 & -1 & -1 & 2 & 1 \\
0 & 0 & 1 & -1 & 1 \\
0 & 0 & -2 & 2 & -2
\end{array}\right] \stackrel{L_{3}+2 L_{2}}{\longrightarrow}\left[\begin{array}{rrrr|r}
1 & -1 & -1 & 2 & 1 \\
0 & 0 & 1 & -1 & 1 \\
0 & 0 & 0 & 0 & 0
\end{array}\right],
$$

Para obtermos a forma escalonada reduzida, devemos zerar a posição acima do 1 líder da segunda linha, terceira coluna. Aplicando a operação elementar $L_{1} \rightarrow L_{1}+L_{2}$ e obtemos

$$
\left[\begin{array}{rrrr|r}
1 & -1 & 0 & 1 & 2 \\
0 & 0 & 1 & -1 & 1 \\
0 & 0 & 0 & 0 & 0
\end{array}\right]
$$

cujo sistema associado é

$$
S^{\prime}:\left\{\begin{array}{l}
w-x+z=2 \\
y-z=1
\end{array}\right.
$$

Resolvendo S', de trás para a frente, obtemos $y=1+z$ e $w=2+x-z$, ou seja, o conjunto solução de S' e, consequentemente de S é

$$
\{(2+x-z, x, 1+z, z) ; x, z \in \mathbb{R}\}
$$

Neste caso, dizemos que $x$ e $z$ são variáveis livres e $y$ e $w$ são variáveis dependentes.

\section{4 - Caracterização de um sistema com relação ao número de solução.}

Finalizaremos este capítulo caracterizando sistemas de equações lineares, com respeito ao número de soluções, ou seja, mostraremos que:

Para um sistema de equações lineares S com coeficientes reais, temos uma e somente uma das possibilidades abaixo verdadeiras:

(a) S admite uma única solução, ou seja, é possível e determinado.

(b) S admite infinitas soluções, ou seja, é possível e indeterminado.

(c) S não admite solução, ou seja, é impossível. 
Consideremos o sistema de equações lineares

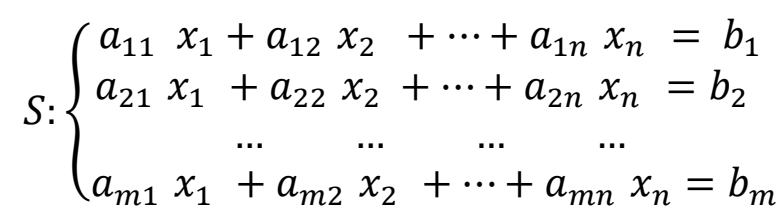

Com matriz aumentada

$$
\left[\begin{array}{cccc|c}
a_{11} & a_{12} & \cdots & a_{1 n} & b_{1} \\
a_{21} & a_{22} & \cdots & a_{2 n} & b_{2} \\
\vdots & \vdots & \cdots & \vdots & \vdots \\
a_{m 1} & a_{m 2} & \cdots & a_{m n} & b_{m}
\end{array}\right]
$$

O sistema de equações lineares

$$
\left\{\begin{array}{c}
a_{11} x_{1}+a_{12} x_{2}+\cdots+a_{1 n} x_{n}=0 \\
a_{21} x_{1}+a_{22} x_{2}+\cdots+a_{2 n} x_{n}=0 \\
\cdots \quad \cdots \quad \cdots \quad \cdots \\
a_{m 1} x_{1}+a_{m 2} x_{2}+\cdots+a_{m n} x_{n}=0
\end{array}\right.
$$

é dito ser o sistema linear homogêneo associado ao sistema S.

Novamente, para facilitar a maneira de escrever, usaremos a representação dos sistemas lineares na forma de produto de matrizes. O sistema $\mathrm{S}$ será escrito na forma $\mathbf{A X}=\mathbf{b}$, onde

$$
\mathbf{A}=\left[\begin{array}{cccc}
a_{11} & a_{12} & \cdots & a_{1 n} \\
a_{21} & a_{22} & \cdots & a_{2 n} \\
\vdots & \vdots & \vdots & \vdots \\
a_{m 1} & a_{m 2} & \cdots & a_{m n}
\end{array}\right], \quad \mathbf{X}=\left[\begin{array}{c}
x_{1} \\
x_{2} \\
\vdots \\
x_{n}
\end{array}\right], \quad \text { e } \quad \mathbf{b}=\left[\begin{array}{c}
b_{1} \\
b_{2} \\
\vdots \\
b_{m}
\end{array}\right]
$$

e, o sistema linear homogêneo associado será $\mathbf{A X}=\mathbf{O}$, onde $\mathbf{O}$ é a matriz nula $m X 1$.

\subsection{1- Teorema}

Seja S um sistema de equações lineares como acima. Então apenas uma das seguintes afirmações é verdadeira:

(I) S admite uma única solução, ou seja, é possível e determinado.

(II) S admite infinitas soluções, ou seja, é possível e indeterminado. 
(III) S não admite solução, ou seja, é impossível.

Demonstração: É imediato que, ou S é possível ou impossível. Se S for impossível, vale (III). Se $\mathrm{S}$ for um sistema possível, temos que mostrar que, vale (I) ou (II).

Se S admite uma única solução, temos (I). Suponhamos então que $\mathrm{S}$ admite mais de uma solução. Temos que mostrar que, então S admite infinitas soluções, i.é, vale (II).

Sejam $u_{1}=\left(k_{1}, \ldots, k_{n}\right)$ e $u_{2}=\left(s_{1}, \ldots, s_{n}\right)$ duas soluções distintas de S. Então $u_{0}=\left(k_{1}-s_{1}, \ldots, k_{n}-s_{n}\right)=u_{1}-u_{2}$ é uma solução não nula do sistema linear homogêneo associado à $\mathrm{S}$, ou seja, se a forma matricial de $\mathrm{S}$ é $A X_{0}=b$, então $A u_{0}=0$, onde

$$
u_{o}=\left[\begin{array}{c}
k_{1}-s_{1} \\
\vdots \\
k_{n}-s_{n}
\end{array}\right] .
$$

Claramente $a u_{0}$ é também solução de $\mathrm{AX}=\mathrm{O}$, para todo $a \in \mathbb{R}$. Assim $u=u_{1}+$ $a u_{0}$, para cada $a \in \mathbb{R}$, é uma solução de $\mathrm{S}$, pois $A u=A\left(u_{1}+a u_{0}\right)=A u_{1}+A\left(a u_{0}\right)=b+$ $O=b$. Portanto $\mathrm{S}$ admite infinitas soluções.

Observamos que, no Teorema 1.4.1, mostramos que dado um sistema de equações lineares, não ocorre duas das afirmações acima ao mesmo tempo. Dos Exemplos 1.2.2, 1.2.3 e 1.2.4 tem-se que ocorrem as três afirmações. 


\section{Capítulo II}

\section{Aplicações de Sistemas de Equações Lineares}

O objetivo neste capítulo é mostrarmos como utilizar o conteúdo de Sistemas de Equações Lineares na solução de problemas do dia a dia apresentado em diferentes tipos de situações. Os métodos utilizados para a interpretação matemática e o equacionamento em cada situação apresentam características comuns, embora sejam situações de naturezas diferentes.

\section{1 - Redes Elétricas}

Circuitos elétricos é um assunto trabalhado na $3^{\mathrm{a}}$ série do Ensino Médio com o objetivo de mostrar que os fenômenos elétricos encontram-se presentes no cotidiano de todos, pois há uma infinidade de aparelhos e equipamentos cujo funcionamento depende de correntes elétricas.

Discutiremos as leis básicas dos circuitos elétricos e mostraremos como estas leis podem ser usadas para obtermos os sistemas de equações lineares cujas soluções fornecem as correntes que fluem num dado circuito elétrico.

Os circuitos elétricos consistem dos seguintes componentes:
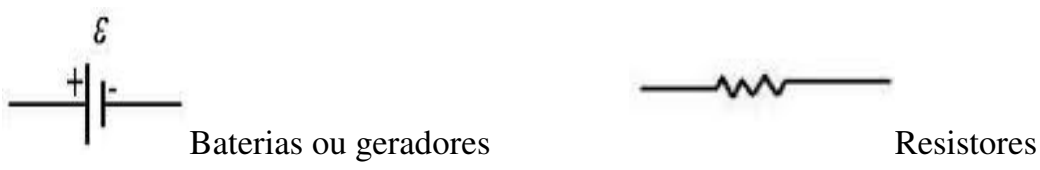

Figura 2.1.a

Os geradores elétricos, tais como as baterias, criam correntes num circuito elétrico e os resistores como as lâmpadas elétricas limitam as magnitudes das correntes. 
Existem três grandezas físicas usadas no estudo de circuitos elétricos: o potencial elétrico $(\mathrm{E})$ medido em volts $(\mathrm{V})$, a resistência $(\mathrm{R})$ medida em ohms $(\Omega)$ e a intensidade de corrente (I) medida em ampères (A).

O potencial elétrico é associado com dois pontos de um circuito elétrico e na prática é medido conectando estes dois pontos a um aparelho chamado voltímetro. Por exemplo, uma pilha AA comum é classificada como tendo $1,5 \mathrm{~V}$, o que significa que esta é a diferença de potencial elétrico entre seus terminais positivo e negativo.

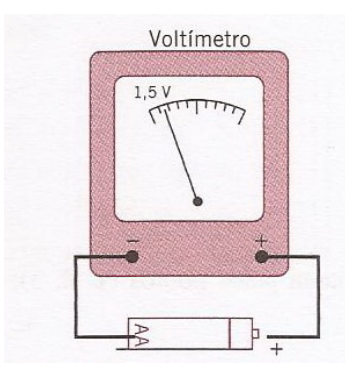

Figura 2.1.b

Num circuito elétrico, o potencial elétrico entre dois pontos é chamado de diferença de potencial ou queda de tensão entre estes dois pontos. Como veremos, as intensidades de correntes e as quedas de tensão podem ser tanto positivas quanto negativas.

O fluxo da corrente num circuito elétrico é governado por três princípios básicos:

1. A lei do $\mathbf{O h m}$ - A diferença de potencial através de um resistor é o produto da corrente que passa por ele e a resistência, ou seja, E = I R.

2. A Lei de Corrente de Kirchhoff - A soma algébrica das correntes fluindo para dentro de qualquer ponto de um circuito elétrico é igual à soma algébrica das correntes fluindo para fora do ponto.

3. A Lei de Voltagem de Kirchhoff - Em torno de qualquer circuito fechado (também chamado de malha), a soma algébrica das diferenças de potencial é zero.

\section{Encontrando correntes num circuito.}

Encontre as correntes $I_{1}, I_{2}$ e $I_{3}$ do circuito abaixo: 


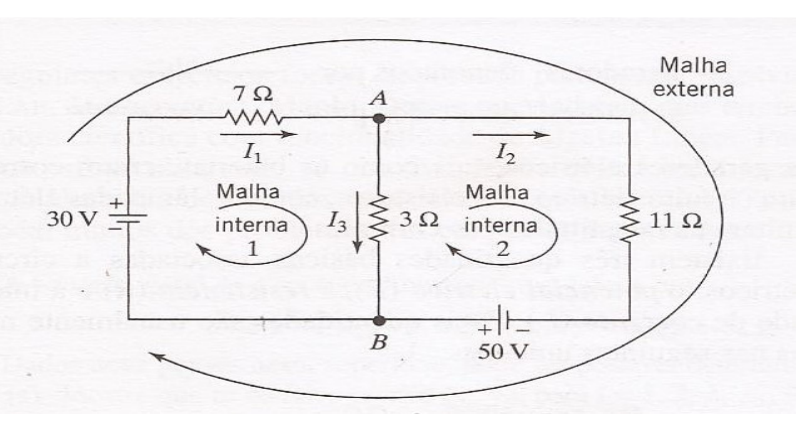

Figura 2.1.c

As direções dos fluxos para as correntes $I_{1}, I_{2} e I_{3}$ (marcadas por flechas) foram tomadas arbitrariamente. Se alguma destas correntes for negativa é por que, na realidade, flui no sentido oposto ao selecionado.

Aplicando a Lei de Corrente de Kirchhoff aos pontos $A$ e $B$, obtemos:

$$
\begin{cases}I_{1}=I_{2}+I_{3} & (\text { Ponto } A) \\ I_{3}+I_{2}=I_{1} & (\text { Ponto } B)\end{cases}
$$

Como as duas equações representam a mesma equação linear $I_{1}-I_{2}-I_{3}=0$, precisamos de mais duas equações para determinar $I_{1}, I_{2}$ e $I_{3}$ de modo único. Estas equações serão obtidas com a Lei de Voltagem de Kirchhoff.

Para aplicar a Lei de Voltagem de Kirchhoff a um circuito fechado, selecione um sentido positivo em torno do circuito (digamos, sentido horário) e faça a seguinte convenção de sinais:

- Uma corrente passando por um resistor produz uma diferença de potencial positiva se flui no sentido positivo do circuito e uma diferença de potencial negativa se flui no sentido negativo do circuito.

- Uma corrente passando por um capacitor produz uma diferença de potencial positiva se o sentido positivo do circuito é de + para - e uma diferença de potencial negativa se o sentido positivo do circuito é de - para + .

Aplicando a Lei de Voltagem de Kirchhoff e a Lei de Ohm à malha interna 1 e 2 da Figura 2.1.c, obtemos respectivamente

$$
7 I_{1}+3 I_{3}-30=0 \quad \text { e } \quad 11 I_{2}-3 I_{3}-50=0
$$

Combinando estas equações obtemos o sistema linear: 


$$
S:\left\{\begin{array}{l}
I_{1}-I_{2}-I_{3}=0 \\
7 I_{1}+3 I_{3}=30 \\
11 I_{2}-3 I_{3}=50
\end{array}\right.
$$

A matriz aumentada de $\mathrm{S}$ é:

$$
\left[\begin{array}{rrr|r}
1 & -1 & -1 & 0 \\
7 & 0 & 3 & 30 \\
0 & 11 & -3 & 50
\end{array}\right]
$$

Resolvendo este sistema linear pelo método de eliminação gaussiana, temos:

$$
\begin{gathered}
{\left[\begin{array}{rrr|r}
1 & -1 & -1 & 0 \\
7 & 0 & 3 & 30 \\
0 & 11 & -3 & 50
\end{array}\right] \stackrel{-7 L_{1}+L_{2}}{\longrightarrow}\left[\begin{array}{rrr|r}
1 & -1 & -1 & 0 \\
0 & 7 & 10 & 30 \\
0 & 11 & -3 & 50
\end{array}\right] \stackrel{\frac{1}{7} L_{2}}{\longrightarrow}\left[\begin{array}{rrr|r}
1 & -1 & -1 & 0 \\
0 & 1 & \frac{10}{7} & \frac{30}{7} \\
0 & 11 & -3 & 50
\end{array}\right]} \\
{\left[\begin{array}{rrr|r}
1 & -1 & -1 & 0 \\
0 & 1 & \frac{10}{7} & \frac{30}{7} \\
0 & 11 & -3 & 50
\end{array}\right] \stackrel{-11 L_{2}+L_{3}}{\longrightarrow}\left[\begin{array}{rrr|r}
1 & -1 & -1 & 0 \\
0 & 1 & \frac{10}{7} & \frac{30}{7} \\
0 & 0 & \frac{-131}{7} & \frac{20}{7}
\end{array}\right] \stackrel{\frac{-7}{131} L_{3}}{\longrightarrow}\left[\begin{array}{rrr|r}
1 & -1 & -1 & 0 \\
0 & 1 & \frac{10}{7} & \frac{30}{7} \\
0 & 0 & 1 & \frac{-20}{131}
\end{array}\right] .}
\end{gathered}
$$

Esta última matriz está na forma escalonada, cujo sistema correspondente é:

$$
\left\{\begin{array}{l}
I_{1}-I_{2}-I_{3}=0 \\
I_{2}+\frac{10}{7} I_{3}=\frac{30}{7} \\
I_{3}=\frac{-20}{131}
\end{array}\right.
$$

Assim temos, $\quad I_{1}=\frac{570}{131} A, \quad I_{2}=\frac{590}{131} A \quad$ e $\quad I_{3}=\frac{-20}{131} A$.

Observe que $I_{3}$ é negativo, o que significa que esta corrente flui no sentido oposto ao indicado na Figura 2.1.c.

Para resolvermos o sistema usando o método de Gauss- Jordan devemos reduzir a matriz aumentada à forma escalonada por linhas, isto é:

$$
\left[\begin{array}{rrr|r}
1 & -1 & -1 & 0 \\
0 & 1 & \frac{10}{7} & \frac{30}{7} \\
0 & 0 & 1 & \frac{-20}{131}
\end{array}\right] \stackrel{\frac{-10}{7} L_{3}+L_{2}}{\longrightarrow}\left[\begin{array}{ccc|c}
1 & -1 & 0 & \frac{-20}{131} \\
0 & 1 & 0 & \frac{590}{131} \\
0 & 0 & 1 & \frac{-20}{131}
\end{array}\right] \stackrel{L_{2}+L_{1}}{\longrightarrow}\left[\begin{array}{ccc|c}
1 & 0 & 0 & \frac{570}{131} \\
0 & 1 & 0 & \frac{590}{131} \\
0 & 0 & 1 & \frac{-20}{131}
\end{array}\right],
$$

obtendo a única solução do sistema, $\quad I_{1}=\frac{570}{131} A, \quad I_{2}=\frac{590}{131} A \quad$ e $\quad I_{3}=\frac{-20}{131} A$. 


\section{2- Balanceamento de Equações Químicas.}

Balanceamento de equações químicas é um assunto trabalhado na $1^{\mathrm{a}}$ série do Ensino Médio com o objetivo de que o aluno seja capaz de entender que equação química é uma forma de descrever uma reação química e como podemos fazer o balanceamento da equação, usando o estudo de resolução de sistemas lineares.

Numa equação química é sempre importante verificar se o número de átomos de cada elemento é o mesmo em ambos os lados da equação, ou seja, se ela está balanceada. Os números que colocamos antes dos símbolos são denominados coeficientes estequiométricos. Esses coeficientes devem ser os menores inteiros possíveis, pois não dá para imaginar 1/2 molécula de algum elemento químico. Note que nunca haverá uma única equação balanceada para uma reação, já que todo múltiplo inteiro positivo de uma equação balanceada será também uma equação balanceada. Assim, usualmente procuramos a equação balanceada mais simples para uma reação.

Para isso vamos analisar a combustão da gasolina. A gasolina é uma mistura de elementos químicos chamados hidrocarbonetos, mas o composto predominante é o $C_{8} H_{18}$, a combustão completa da gasolina acontece quando reage com o gás oxigênio resultando em gás carbônico e água, então,

$$
\mathrm{C}_{8} \mathrm{H}_{18}+\mathrm{O}_{2} \rightarrow \mathrm{CO}_{2}+\mathrm{H}_{2} \mathrm{O}
$$

Agora, se faz necessário balancear a equação, e para isso vamos utilizar sistemas de equações lineares. Chamando as quantidades de cada molécula da fórmula de $x, y, w e z$, temos:

$$
x \mathrm{C}_{8} \mathrm{H}_{18}+y \mathrm{O}_{2} \rightarrow w \mathrm{CO}_{2}+z \mathrm{H}_{2} \mathrm{O} .
$$

Para os átomos de carbono: $8 x=w$.

Para os átomos de hidrogênio: $18 x=2 z \rightarrow 9 x=z$.

Para os átomos de oxigênio: $2 y=2 w+z$.

Obtemos então o seguinte sistema de equações lineares:

$$
S:\left\{\begin{array}{l}
8 x-w=0 \\
9 x-z=0 \\
2 y-2 w-z=0
\end{array}\right.
$$

Note que, $\mathrm{S}$ tem 3 equações e 4 variáveis, o que implica que $\mathrm{S}$ é possível e indeterminado, ou seja, admite infinitas soluções. 
Considerando a matriz aumentada de $\mathrm{S}$

$$
\left[\begin{array}{rrrr|r}
8 & 0 & -1 & 0 & 0 \\
9 & 0 & 0 & -1 & 0 \\
0 & 2 & -2 & -1 & 0
\end{array}\right]
$$

e aplicando o Método de eliminação de Gauss, obtemos a matriz escalonada correspondente

$$
\left[\begin{array}{cccc|c}
1 & 0 & \frac{-1}{8} & 0 & 0 \\
0 & 1 & -1 & \frac{-1}{2} & 0 \\
0 & 0 & 1 & \frac{-8}{9} & 0
\end{array}\right],
$$

cujo sistema associado é:

$$
S^{\prime}:\left\{\begin{array}{l}
x-\frac{1}{8} w=0 \\
y-w-\frac{1}{2} z=0 \\
w-\frac{8}{9} z=0
\end{array}\right.
$$

Assim, o conjunto das soluções de $S$, que é o conjunto das soluções de $S$ ' é $\left\{\left(\frac{1}{8} w, \frac{25}{16} w, \frac{9}{8} w, w\right) ; w \in \mathbb{R}\right\}$

Para a solução mais simples os coeficientes estequiométricos devem ser os menores inteiros que satisfazem todas as equações portanto, temos que, $x=2, y=25, z=18$ e $w=16$ é a solução da equação e, consequentemente, a equação balanceada é:

$$
2 \mathrm{C}_{8} \mathrm{H}_{18}+25 \mathrm{O}_{2} \rightarrow 16 \mathrm{CO}_{2}+18 \mathrm{H}_{2} \mathrm{O}
$$

\section{3 - Controle do fluxo de veículos nas ruas de mão única no horário do rush.}

Através desta aplicação, que pode ser ministrada para a segunda série do Ensino Médio, podemos determinar a média do número de veículos em cada cruzamento em um determinado horário.

Durante o dia é fácil observar que há vários fluxos de veículos em determinados pontos da cidade. Assim, podemos fazer um trabalho de conscientização dos motoristas para que nos períodos de rush eles não cometam excessos. Usando o mínimo necessário da buzina, ouvindo um som a um volume audível somente aos ocupantes do carro, principalmente em locais próximos às escolas, hospitais, creches, asilos entre outros. 
Vejamos um exemplo. Suponha que uma determinada cidade tem dois conjuntos de ruas de mão única que se cruzam como mostra a figura abaixo:

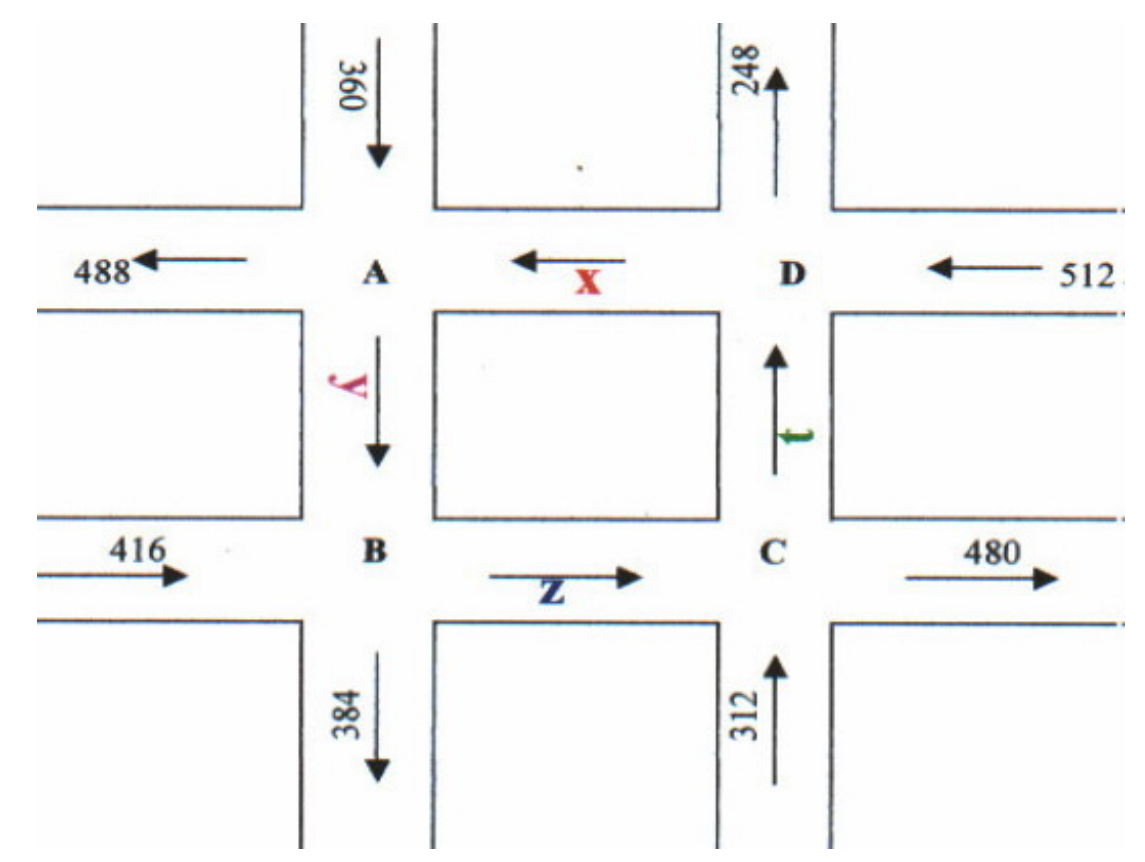

Disponível em:

http://www.unifra.br/eventos/jornadaeducacao2006/2006/pdf/artigos/matem\%C3\%A1tica/A\%20MODELAGEM\%20MATE M\%C3\%81TICA\%20NO\%20ENSINO\%20DE\%20MATRIZES.pdf

Queremos determinar a média do número de veículos por hora que entram e saem dessa seção durante o horário do rush. Para tanto necessitamos determinar a quantidade de veículos entre cada um dos quatro cruzamentos. Observando que o número de entrada de veículos é igual ao número de saída e que o fluxo tem o mesmo sentido das setas indicadas na figura, obtemos:

$$
\begin{aligned}
& 360+x=488+y, \quad(\text { cruzamento } A) \\
& 416+y=384+z, \quad(\text { cruzamento } B) \\
& 312+z=480+t, \quad(\text { cruzamento } C) \\
& 512+t=248+x, \quad(\text { cruzamento } D)
\end{aligned}
$$


Temos então o seguinte sistema de equações lineares:

$$
\left\{\begin{array}{l}
x-y=128 \\
y-z=-32 \\
z-t=168 \\
-x+t=-264
\end{array}\right.
$$

Cuja matriz aumentada é

$$
\left[\begin{array}{rrrr|r}
1 & -1 & 0 & 0 & 128 \\
0 & 1 & -1 & 0 & -32 \\
0 & 0 & 1 & -1 & 168 \\
-1 & 0 & 0 & 1 & -264
\end{array}\right]
$$

Aplicando o método de Eliminação de Gauss obtemos a matriz escalonada:

$$
\left[\begin{array}{rrrr|r}
1 & -1 & 0 & 0 & 128 \\
0 & 1 & -1 & 0 & -32 \\
0 & 0 & 1 & -1 & 168 \\
0 & 0 & 0 & 0 & 0
\end{array}\right],
$$

com o seguinte sistema linear correspondente:

$$
\left\{\begin{array}{c}
x-y=128 \\
y-z=-32 \\
z-t=168
\end{array}\right.
$$

Este sistema é compatível e possui uma variável livre, portanto possui infinitas soluções. Fazendo $t$ como variável livre, obtemos o conjunto solução

$$
\{(264+t, 136+t, 168+t, t) t \in \mathbb{N}\} .
$$

Para determinar o número de veículos em cada cruzamento devemos conhecer o valor de $t$, que é o número de veículos entre os cruzamentos $C$ e $D$. Para $t=200$, temos:

$$
\begin{aligned}
& z=368 \text { (número de veículos entre os cruzamentos } B \text { e } C \text { ). } \\
& y=236 \text { (número de veículos entre os cruzamentos } A \text { e } B \text { ). } \\
& x=364 \text { (número de veículos entre os cruzamentos } A \text { e } D \text { ). }
\end{aligned}
$$




\section{Capítulo III}

\section{Sequência Didática - Condicionamento Físico}

A sequência didática descrita a seguir tem como objetivo demonstrar que a matemática é uma ciência que não se limita a um ambiente restrito sem aplicação ou finalidade, ela é ampla em sua magnitude e todas as outras ciências estão diretamente relacionadas com ela. Para a aplicação dessa sequência didática é proposta uma situação problema contextualizada visando desenvolver nos alunos o caráter investigativo e levar o professor a refletir sobre as estratégias que possibilitam uma articulação entre as ações didáticas e o gerir do conhecimento autônomo.

As atividades de caráter investigativo implicam, inicialmente, a proposição de situações-problemas, que, então orientam e acompanham todo o processo de investigação. Nesse contexto o professor desempenha o papel de guia e de orientador das atividades, é ele quem propõe e discute questões, contribui para o planejamento da investigação dos alunos, orienta o levantamento de evidências e explicações teóricas, possibilita a discussão e a argumentação entre os estudantes, introduz conceitos e promove a sistematização do conhecimento. Consequentemente, o professor oportuniza, de forma significativa, a vivência de experiências pelos estudantes, permitindo-lhes, assim, a construção de novos conhecimentos a cerca do que está sendo investigado.

O aluno como construtor de seu próprio conhecimento é um assunto recente que tem permeado o âmbito escolar. Logo, o principal objetivo dessa sequência didática foi o estudo de Sistemas de Equações Lineares, através de situações contextualizadas.

De acordo com os PCN (1998, p.37),

as necessidades cotidianas fazem com que os alunos desenvolvam capacidades de natureza prática para lidar com a atividade matemática, o que lhes permite reconhecer problemas, buscar e selecionar 
informações, tomar decisões. Quando essa capacidade é potencia-

lizada pela escola, a aprendizagem apresenta melhor resultado.

Nesse sentido, foi proposto aos alunos do segundo ano do Ensino Médio que a partir de uma pesquisa em seu bairro investigassem sobre o hábito das práticas de atividades físicas dos habitantes.

Desejava-se que o aluno entendesse a importância da prática de atividade física para uma melhor qualidade de vida, pois é uma aliada imprescindível para alcançar uma boa forma física e sua prática deve ser desenvolvida de uma forma prazerosa e contínua ao longo de toda a vida.

Pesquisas mostram que pessoas que incluem atividades físicas no seu programa de emagrecimento têm menor chance de recuperar o peso perdido do que as que só mudaram a dieta. Além de promover o controle de peso, a atividade física melhora sua força e flexibilidade, diminui o risco de enfermidade cardíaca, ajuda a controlar a pressão sanguínea e diabetes e ainda pode melhorar a sensação de bem-estar e diminuir o estresse.

A preocupação de promover e manter a saúde devem ser ressaltados para a população pesquisada, que, cada vez mais, necessita, em sua rotina diária, da prática de exercícios físicos regulares para combater os efeitos nocivos da vida sedentária.

Também se pretendia que eles estivessem aptos a fazer comparativos entre a quantidade de horas para cada atividade física e a quantidade de calorias gastas para cada tipo de atividade física em cada faixa etária.

\subsection{Descrições, metodologia e aplicação.}

Inicialmente foi proposto aos alunos que se organizassem em grupos e que cada grupo entrevistasse cinco pessoas de diferentes estatus, profissionais liberais, professores, donas de casa, estudantes, comerciantes, balconistas e outros, de diferentes faixas etárias, jovens e adultos, sobre a regularidade da prática de atividade física bem como a respeito do que eles sabem sobre os benefícios dessa prática.

Com os dados coletados efetuou-se uma média da quantidade de horas gastas por semana com cada atividade entre os jovens e os adultos, esses dados foram organizados em uma tabela como no exemplo a seguir. 


\begin{tabular}{|l|l|l|}
\hline \multicolumn{2}{|l|}{ Atividade: } \\
\hline \multirow{2}{*}{$\begin{array}{l}\text { Dia da } \\
\text { semana }\end{array}$} & \multicolumn{2}{|c|}{ (Horas / Dia) } \\
\cline { 2 - 3 } Segens & Adultos \\
\hline Segunda-feira & & \\
\hline Quarta-feira & & \\
\hline Sexta-feira & & \\
\hline
\end{tabular}

Tabela 1: Quantidade de horas/dia para cada atividade física

\begin{tabular}{|l|c|c|}
\hline \multicolumn{3}{|c|}{ Atividade: Caminhar (3 km/h) } \\
\hline $\begin{array}{l}\text { Dia da } \\
\text { semana }\end{array}$ & \multicolumn{2}{|c|}{ (Horas / Dia) } \\
\cline { 2 - 3 } Sovens & Adultos \\
\hline Segunda-feira & 1 & 1 \\
\hline Quarta-feira & 1,5 & 1 \\
\hline Sexta-feira & 1 & 1 \\
\hline
\end{tabular}

Tabela2: Quantidade de horas/dia para caminhada

\begin{tabular}{|l|c|c|}
\hline \multicolumn{2}{|l|}{ Atividade: Correr (9 km/h) } \\
\hline \multirow{2}{*}{$\begin{array}{l}\text { Dia da } \\
\text { semana }\end{array}$} & \multicolumn{2}{|c|}{ (Horas / Dia) } \\
\cline { 2 - 3 } Jovens & Adultos \\
\hline Segunda-feira & 2 & 1 \\
\hline Quarta-feira & 1 & 0,5 \\
\hline Sexta-feira & 1 & 0,5 \\
\hline
\end{tabular}

Tabela 3: Quantidade de horas/dia para corrida 


\begin{tabular}{|l|c|c|}
\hline \multicolumn{2}{|c|}{ Atividade: Andar de bicicleta (9 km/h) } \\
\hline $\begin{array}{l}\text { Dia da } \\
\text { semana }\end{array}$ & \multicolumn{2}{|c|}{ (Horas / Dia) } \\
\cline { 2 - 3 } Jovens & Adultos \\
\hline Segunda-feira & 0,5 & 0,5 \\
\hline Quarta-feira & 0,5 & 0,5 \\
\hline Sexta-feira & 1 & 1 \\
\hline
\end{tabular}

Tabela 4:Quantidade de horas/dia para andar de bicicleta

Depois de elaborada a pesquisa desejava-se descobrir quantas calorias com cada tipo de atividade deveriam ser gastas por uma pessoa que pretende perder peso fazendo atividades físicas.

Para isso foi tomado como exemplo duas pessoas com mesmo peso $(69 \mathrm{~kg}) \mathrm{de}$ diferentes faixas etárias. Ana que é jovem e deseja perder 0,52 quilogramas (aproximadamente) semanalmente e Célia, adulta que pretende reduzir sua massa corporal para estar dentro da normalidade de acordo com o IMC (Índice de Massa Corporal). Ana sabe que é preciso gastar 3.953,50 calorias por semana, pois 7.700 calorias equivalem a um quilograma.

A tabela abaixo contêm as horas por dia que Ana dedicará para cada tipo de atividade física:

\begin{tabular}{|c|c|c|c|}
\hline Dia da semana & $\begin{array}{c}\text { Caminhar } \\
\mathbf{( 3 k m / h )} \\
(\text { horas/dia) }\end{array}$ & $\begin{array}{c}\text { Correr } \mathbf{( 9 k m / h ) ~} \\
\text { (horas/dia) }\end{array}$ & $\begin{array}{c}\text { Andar de } \\
\text { bicicleta } \mathbf{( 9 ~} \mathbf{~ k m} / \mathbf{h}) \\
\text { (horas/dia) }\end{array}$ \\
\hline Segunda-feira & 1 & 2 & 0,5 \\
\hline Quarta-feira & 1,5 & 1 & 0,5 \\
\hline Sexta-feira & 1 & 1 & 1 \\
\hline
\end{tabular}

Considerando-se que Ana quer gastar na segunda feira 1.665 calorias, na quarta- feira 1.121,5 calorias e na sexta- feira 1.167 calorias, quantas calorias são gastas com cada atividade?

Diante de todos esses questionamentos os alunos foram estimulados a utilizarem a linguagem matemática para expressar as condições descritas na situação problema destacando 
elementos importantes para a compreensão e interpretação dos resultados. E com isso chamaram de $x$ a quantidade de calorias por hora de atividade que são gastas caminhando a $3 \mathrm{~km} / \mathrm{h}, y$ correndo a $9 \mathrm{~km} / \mathrm{h}$ e $z$ andando de bicicleta a $9 \mathrm{~km} / \mathrm{h}$.

Com os dados acima, obtemos o seguinte sistema de equações lineares:

$$
\left\{\begin{array}{l}
1 x+2 y+0,5 z=1665 \\
1,5 x+1 y+0,5 z=1121,5 \\
1 x+1 y+1 z=1167
\end{array}\right.
$$

Neste momento os alunos apresentaram algumas estratégias para obtenção da solução do sistema proposto, eles foram incentivados a falar sobre as estratégias que pensaram. Aqui o objetivo principal era resgatar o conhecimento prévio deles, auxiliando-os na compreensão do conteúdo deste assunto, além de contribuir para que percebessem que uma estratégia bastante adequada para resolver esta situação proposta é usar o Método de Eliminação de Gauss para resolver o sistema linear.

A matriz aumentada do sistema é:

$$
\left[\begin{array}{rrr|r}
1 & 2 & 0,5 & 1665 \\
1,5 & 1 & 0,5 & 1121,5 \\
1 & 1 & 1 & 1167
\end{array}\right]
$$

e, desenvolvendo pelo Método de Eliminação de Gauss, obtemos a matriz escalonada correspondente:

$$
\left[\begin{array}{rrr|r}
1 & 1 & 1 & 1167 \\
0 & 1 & -0,5 & 498 \\
0 & 0 & -1,25 & -380
\end{array}\right]
$$

Assim a única solução do sistema é $(213,650,304)$ e, podemos concluir que Ana, com $69 \mathrm{~kg}$, necessita perder 213 calorias por hora ao caminhar a $3 \mathrm{~km} / \mathrm{h}, 650$ calorias ao correr a 9 $\mathrm{km}$ por hora e 304 calorias por hora ao andar de bicicleta a $9 \mathrm{~km} / \mathrm{h}$.

Já entre os adultos a tabela que representa a quantidade de horas por dia, dedicada para cada atividade física está representada a seguir:

\begin{tabular}{|c|c|c|c|}
\hline Dia da semana & $\begin{array}{c}\text { Caminhar } \\
(\mathbf{3} \mathbf{~ k m} / \mathbf{h}) \\
(\mathbf{h o r a s} / \mathbf{d i a})\end{array}$ & $\begin{array}{c}\text { Correr }(\mathbf{9} \mathbf{~ k m} / \mathbf{h}) \\
(\text { horas/dia) }\end{array}$ & $\begin{array}{c}\text { Andar de } \\
\text { bicicleta } \mathbf{( 9} \mathbf{~ k m} / \mathbf{h}) \\
(\text { horas/dia) }\end{array}$ \\
\hline Segunda-feira & 1 & 1 & 0,5 \\
\hline Quarta-feira & 1 & 0,5 & 0,5 \\
\hline Sexta-feira & 1 & 0,5 & 1 \\
\hline
\end{tabular}


Visto que a quantidade de horas disponível para a prática de atividade física entre os adultos é menor e, portanto seguindo a mesma regra que para os jovens foi possível determinar quanto tempo Célia que tem 1,58 m de altura e pesa $69 \mathrm{~kg}$ levará para ter o seu IMC (Índice de Massa Corporal) considerado normal, sendo este entre 46,1 kg e 62,1 kg.

Considerando que são gastas 213 calorias ao caminhar a $3 \mathrm{~km} / \mathrm{h}, 650$ calorias ao correr a $9 \mathrm{~km} / \mathrm{h}$ e 304 calorias ao andar de bicicleta a $9 \mathrm{~km} / \mathrm{h}$, um adulto irá gastar 3.237 calorias semanalmente e isto representa aproximadamente $0,42 \mathrm{~kg}$ e para perder no mínimo $6,9 \mathrm{~kg}$ e no máximo $22,9 \mathrm{~kg}$ e estar com seu IMC considerado normal, são necessárias aproximadamente de 17 a 55 semanas.

\section{2 - Considerações finais}

Com essa sequência didática foi possível observar um maior interesse, entusiasmo e motivação dos alunos pelas aulas de matemática, pois observaram que a matemática está presente em nosso cotidiano.

Os conteúdos abordados através de situações problemas possibilita um conhecimento matemático mais significativo, assim é possível mostrar aos alunos que existem diferentes aplicabilidades, e com isso contribuir para sua formação integral para a vida e para o trabalho.

A aplicação de situações reais com o desenvolvimento do conteúdo de sistemas de equações lineares para a interpretação e análise fez com que os alunos enxergassem o quanto à matemática é importante e faz parte do nosso dia-a-dia.

Conclui-se que com esta metodologia nas aulas de Matemática além de servir como motivação para introduzir novas ideias propicia, também, a compreensão e interpretação de um problema real onde o aluno está inserido e faz parte deste processo como cidadão.

Desta forma, o ensino da Matemática cumpre a sua função de contribuir na formação do indivíduo, tratando de assuntos e questões do dia-a-dia, com a intenção de mostrar, conhecer e até mesmo alertar. 


\section{Referências Bibliográficas}

[1] A. Hefez, C. S. Fernades; Introdução a Álgebra Linear; Coleção PROFMAT, SBM, 2012.

[2] Anton, H., Rorres, C.: Álgebra Linear com Aplicações; Bookman, 2001.

[3] A. dos Santos Machado; Temas e Metas Vol.3; Atual Editora, 1986

[4] C. A. Callioli, H.H. Domingues, R. C. F. Costa; Álgebra Linear e Aplicações; Atual Editora, 1995.

[5] G. Iezzi, S. Hazzan; Fundamentos de Matemática Elementar (Sequências Matrizes Determinantes Sistemas) Vol.4; Atual Editora, 2006

[6] Proposta Curricular do Estado de São Paulo; Matemática/ coord. Maria Inês Fini.São Paulo: SEE, 2008.

[7] S. Lipschutz, Álgebra Linear; Editora McGraw-Hill do Brasil Ltda, 1978

[8] Y. Y. Baldin, Y.K. S. Furuya, Geometria Analítica para todos e atividades com Octave e GeoGebra; EduFSCar, 2012.

[9] D. Poole, Álgebra Linear; São Paulo: Pioneira Thomson Learning, 2004.

[10] Caderno do Professor de Matemática, Ensino Fundamental; Vol. 3 , $8^{\circ}$ ano.

[11] Caderno do Professor de Matemática, Ensino Médio; Vol. 2, 2ªno.

[12] Brasil, Ministério da Educação. Parâmetros Curriculares Nacionais (PCN’s); Ensino Médio, 2000. 